\title{
Haemolysin-deficient variants of Streptococcus pyogenes and $S$. dysgalactiae subsp. equisimilis may be overlooked as aetiological agents of pharyngitis
}

\author{
KAREN P. DIERKSEN and JOHN R. TAGG \\ Department of Microbiology, University of Otago, PO Box 56, Dunedin, New Zealand
}

\begin{abstract}
Variants of large colony $\beta$-haemolytic Lancefield group A, C and $\mathrm{G}$ streptococci that are non-haemolytic or $\alpha$-haemolytic on sheep blood agar have been detected in clinical specimens due to their enhanced haemolytic activity when grown on a new selective and differential blood agar medium containing colistin, nalidixic acid and $\mathbf{p H}$ 7.5-adjusted PIPES buffer (CNA-P). The large colony Lancefield group $C$ and $G$ isolates were identified as Streptococcus dysgalactiae subsp. equisimilis by API 20 Strep classification and 16S rDNA profiling. The haemolytic activity of these variants on various blood agar media, including CNA-P, was closely similar to that of known streptolysin S-defective mutants of $S$. pyogenes and was blocked by addition of cholesterol, a specific inhibitor of the streptolysin $O$ family of haemolysins. As haemolysin variants could be detected in large numbers in cultures from patients with clinical symptoms of pharyngitis it is suggested that they may function as primary pathogens in such infections. The high frequency with which haemolysin variants were isolated from clinical specimens during a 3-month trial $(3 \%, 13 \%$ and $10 \%$, respectively, of group $A, C$ and $G$ streptococcal isolates) indicated that a substantial proportion of streptococcal infections may go undetected if only conventional sheep blood agar media are used in clinical laboratories for the detection of $\beta$-haemolytic streptococci. As haemolysin variants have been implicated in the development of serious streptococcal sequelae, further investigation of the full extent of their contribution to streptococcal disease is indicated.
\end{abstract}

\section{Introduction}

$\beta$-Haemolytic streptococci of Lancefield groups A, C and $\mathrm{G}$ are commonly present in the human pharynx where they can adopt either a commensal or a pathogenic role. When grown on blood agar media, some of these streptococci typically form small colonies and others form relatively large colonies. The small colony forms belong to the Streptococcus anginosus group, whereas the larger colonies are generally $S$. pyogenes (group A), S. equi subsp. zooepidemicus (group C) or $S$. dysgalactiae supsp. equisimilis (group $\mathrm{C}$ or group $\mathrm{G}$ ). The latter have been associated with a wide variety of diseases including septicaemia, endocarditis, pneumonia and meningitis

Received 22 Nov. 1999; revised version accepted 31 Jan 2000.

Corresponding author: Professor J. R. Tagg (e-mail: john.tagg@stonebow.otago.ac.nz).
[1-4]. There is also the ever-present risk of development of glomerulonephritis [5] or of reactive arthritis $[6,7]$ subsequent to group $\mathrm{C}$ or $\mathrm{G}$ streptococcal infection. Although there have been numerous reports of epidemic group $\mathrm{C}$ and $\mathrm{G}$ streptococcal pharyngitis in adults [8-13], their significance as a cause of pharyngitis in children is less firmly established. Chowdhury et al. [14] considered them not to be significant, whereas Cimolai et al. [15] found large colony isolates to be significant when isolated in moderate to large numbers from a throat culture, and Martin et al. [12] demonstrated that group G streptococci of one DNA fingerprint pattern were isolated from $73 \%$ of cases during a paediatric outbreak of streptococcal pharyngitis.

Considerable research has been undertaken into the relatedness of large colony forms of $\alpha$ - and $\beta$ haemolytic strains of groups $\mathrm{C}, \mathrm{G}$ and $\mathrm{L}$ streptococci of both animal and human origin [16-19]. Recent taxonomic studies have led to these organisms being 
grouped into the subspecies $S$. dysgalactiae subsp. dysgalactiae, comprising $\alpha$-haemolytic and nonhaemolytic strains, and $S$. dysgalactiae subsp. equisimilis which are usually $\beta$-haemolytic, react with Lancefield group A, C, G or L antisera and are most frequently isolated from human infections [18-21].

Although S. pyogenes is generally considered to be $\beta$ haemolytic, non-haemolytic variants have been isolated from disease outbreaks [22]. In one report, a nonhaemolytic variant was associated with an epidemic of pharyngitis which resulted in six cases of rheumatic fever [23]. Non-haemolytic variants of $S$. dysgalactiae supsp. equisimilis have not been reported previously, possibly because such variants may have been presumed to represent isolates of the normally non- $\beta$ haemolytic $S$. dysgalactiae subsp. dysgalactiae.

In the course of evaluating a new selective medium (CNA-P) for isolation of $\beta$-haemolytic streptococci in a clinical laboratory setting [24], group A, C and G haemolytic variants that failed to produce $\beta$-haemolysis on traditional sheep blood agar media, but which were $\beta$-haemolytic on CNA-P were identified. They were examined for production of streptolysins $\mathrm{O}$ and $\mathrm{S}$, and their API-20 Strep and 16S rDNA profiles were determined.

\section{Materials and methods}

\section{Strains, media, and growth conditions}

Laboratory strains were subcultured on Columbia agar base (Gibco, Madison, WI, USA) supplemented with human blood $5 \% \mathrm{v} / \mathrm{v}$ and $\mathrm{CaCO}_{3} \quad 0.1 \%$ (BA-Ca). BA$\mathrm{Ca}$ is used routinely in this laboratory to minimise the effect of acid production on culture viability during strain storage. Strains used in this study included fresh clinical isolates of haemolytic variant $S$. dysgalactiae subsp. equisimilis of Lancefield group C (strains 0726, 1312, 1446 and 3610) and Lancefield group G (strain 0662). Previously described [25] haemolytic variant $S$. pyogenes strains 82-541 (streptolysin S-deficient) and Blackmore (streptolysin O-deficient) were used for comparative purposes. Other controls included the $\beta$ haemolytic $S$. pyogenes M-type 1 strain SF370 (from J. Ferretti, University of Oklahoma, OK, USA), $S$. dysgalactiae subsp. dysgalactiae strain 67 (J. Tagg culture collection) and $S$. equi subsp. zooepidemicus strain 4881 (J. Tagg culture collection). Clinical specimens were plated on to sheep blood agar (SBA), CNA and CNA-P. SBA consisted of Columbia agar base supplemented with defibrinated sheep blood (Life Technologies) $5 \% \mathrm{v} / \mathrm{v}$. CNA-P consisted of Difco Columbia CNA agar (which contains colistin sulphate $10 \mathrm{mg} / \mathrm{L}$ and nalidixic acid $15 \mathrm{mg} / \mathrm{L}$, supplemented with $100 \mathrm{mM}$ PIPES (piperazine-N-N'-bis-[2-ethanesulphonic acid]; Sigma), adjusted to $\mathrm{pH} 7.5$ before autoclaving and then supplemented with sheep blood $5 \% \mathrm{v} / \mathrm{v}$ [24]. CNA lacked PIPES buffer and the $\mathrm{pH}$ was not adjusted before autoclaving. Cholesterol agar was prepared by adding cholesterol $1 \mathrm{~g} / \mathrm{L}$ to CNA-P agar after autoclaving. Agar media were poured to a depth of $4 \mathrm{~mm}$ and stored at $4^{\circ} \mathrm{C}$ in plastic bags until required. Enhanced haemolysis $(\mathrm{EH})$ medium (Difco Columbia blood agar base EH medium; product code 0790) was prepared by Fort Richards Laboratories, New Zealand. The component Bacto Bitone H Plus is responsible for the enhanced $\beta$-haemolytic reactions on this medium.

\section{Clinical specimens}

Throat swabs obtained from patients with clinical symptoms of pharyngitis and referred to Southern Community Laboratories by physicians in the Dunedin area were plated on SBA, CNA and CNA-P and examined for $\beta$-haemolytic streptococci. A total of 361 pharyngeal swabs received during March-May 1999 was tested. Each swab was plated on to SBA, CNA and CNA-P, with the order of plating rotated weekly. The inoculum was streaked into four quadrants for single colony isolation by a standardised procedure [26]. Plates were incubated at $37^{\circ} \mathrm{C}$ in an atmosphere of $\mathrm{CO}_{2}$ $5 \%$ in air and examined after 24 and $48 \mathrm{~h}$. $\beta$ Haemolytic colonies were semi-quantified and the degree of positivity recorded as $1+(10$ or fewer colonies), $2+(11-100$ colonies $), 3+(>100$ colonies, with some present in the third quadrant of the plate) and $4+(\beta$-haemolytic colonies extending into the fourth quadrant).

\section{Identification of isolates}

Initial detection of $\beta$-haemolytic streptococci was based upon their typical haemolysis and colony morphology [26]. Representative $\beta$-haemolytic colonies were subcultured to SBA and their Lancefield group identity was established serologically by latex agglutination (Streptococcal grouping kit, Oxoid) with group A, B, $\mathrm{C}, \mathrm{D}, \mathrm{F}$ and $\mathrm{G}$ antisera. Small colony $\beta$-haemolytic streptococci belonging to the $S$. anginosus group were excluded from this study. The commercial API-20 Strep system (bioMérieux) was used for biochemical classification of isolates. Representative colonies that were $\beta$-haemolytic on CNA-P, but not on SBA and CNA, were subcultured on CNA-P and then re-cultured on to SBA and CNA to confirm the status of their haemolytic activity. Additionally, isolates were serially cultured at least four times on CNA-P to establish whether or not their haemolytic activity was a stable strain characteristic.

\section{Comparison of haemolytic activity with use of various media and incubation conditions}

Pure cultures of haemolytic variants were compared with reference strains of haemolytic group $\mathrm{A}$ and $\mathrm{C}$ streptococci and with known streptolysin S or streptolysin $\mathrm{O}$ mutants for haemolytic activity on various 
media. Subcultures of each strain to be tested were freshly grown on $\mathrm{BA}-\mathrm{Ca}$ at $35^{\circ} \mathrm{C}$ in an atmosphere of $\mathrm{CO}_{2} 5 \%$ in air for $18 \mathrm{~h}$. Samples of these cultures were swabbed on each test medium, streaked for isolated colonies, and a stab was made into the confluent zone for examination of subsurface haemolysis. The media tested were CNA-P, CNA, BA-Ca, SBA and EH agar. Cultures were incubated aerobically, anaerobically and in $\mathrm{CO}_{2} 5 \%$ in air. Colonies were examined for surface and subsurface haemolysis after $18 \mathrm{~h}$ and $48 \mathrm{~h}$. The $\mathrm{pH}$ of the cultures after growth was measured with a surface $\mathrm{pH}$ electrode (Radiometer Copenhagen). Cholesterol-containing medium was used to specifically block streptolysin $\mathrm{O}$ activity.

\section{PCR of $16 S$ rDNA and emm genes}

Nucleic acids were extracted by a boiling lysis method [27] and $1 \mu \mathrm{l}$ of supernate was used in subsequent 50$\mu 1$ PCR reactions. Universal eubacterial primers P0mod and P3mod were used to amplify c. $800 \mathrm{bp}$ at the 5' terminus of the $16 \mathrm{~S}$ rDNA as described by Wilson et al. [28]. Amplicons were purified with a Qiaquick PCR purification kit (Qiagen) and subjected to automated sequencing with an ABI 377 sequencer (Centre for Gene Research, Otago University). DNA-STAR software was used for alignment with $16 \mathrm{~S}$ streptococcal sequences [16].

Emm-typing was accomplished with the group A streptococcus 'all-M' PCR primers as described previously [27, 29]. PCR products were digested with Hae III and HincII restriction endonucleases. Fragment sizes were compared and a representative isolate of each unique restriction profile was subjected to automated sequencing. Homology searches were performed on the GenBank database (BLAST) and the emm database (Centers for Disease Control and Prevention, Atlanta, GA, USA; http://www.cdc.gov/ ncidod/biotech/strep/strepindex.html).

\section{Results}

\section{Isolation of haemolytic variants from clinical specimens}

Of 361 throat cultures examined, 104 (29\%) were positive for large colony $\beta$-haemolytic streptococci and these comprised $64(61 \%)$ Lancefield group A, 30 (29\%) group $\mathrm{C}$ and 10 (10\%) group $\mathrm{G}$ isolates (Table 1).
Seven of these isolates were $\beta$-haemolytic on CNA-P, but not on either CNA or SBA and in each case, the haemolytic variants represented a major portion of the bacterial growth on the plate $(3+$ or $4+$ positivity). Two isolates were group $\mathrm{A}$, four group $\mathrm{C}$ and one group G. Non- $\beta$-haemolytic colonies from the companion CNA and SBA plates were detected that exhibited $\beta$-haemolysis when re-streaked to CNA-P. Conversely, pure isolates of $\beta$-haemolytic colonies from CNA-P were found to be non- $\beta$-haemolytic when re-streaked to CNA and SBA. The group $\mathrm{C}$ and $\mathrm{G}$ isolates were identified as $S$. dysgalactiae supsp. equisimilis by the API 20 Strep system. The two group A variants were isolated early in the study and were inadvertently discarded. A group A haemolytic variant isolated in this laboratory in an earlier study was identified as an M-type $4 S$. pyogenes (data not shown).

\section{Evaluation of haemolytic activity}

The absence of $\beta$-haemolytic activity was examined further by plating pure cultures of the variant isolates and of several previously characterised haemolytic variants on five media containing blood and incubating under different atmospheric conditions (Table 2). Four of the media contained sheep blood and the fifth (BA$\mathrm{Ca}$ incorporated human blood. All four group $\mathrm{C}$ isolates were $\beta$-haemolytic on CNA-P medium and non- $\beta$-haemolytic on CNA, SBA and EH. On BA-Ca there was some slight variation in the haemolytic activity and terminal $\mathrm{pH}$ of these isolates. As both BA$\mathrm{Ca}$ and CNA-P contained additional buffering agents, it was possible that differences in culture $\mathrm{pH}$ were responsible for variations in haemolytic activity, as it is known that the $\mathrm{pH}$ should be maintained between 6.8 and 7.4 to avoid acidic pH-mediated streptolysin $\mathrm{O}$ inactivation or proteolytic degradation by proteases that are most active below pH 6.8 [30]. When the terminal $\mathrm{pH}$ of the group $\mathrm{C}$ streptococcal culture on BA-Ca was $<6.9$ no haemolysis was evident, whereas at $\mathrm{pH}$ values $\geqslant 6.9$ some $\beta$-haemolysis was detected. This observation was consistent with the anticipated haemolytic activity of isolates that produced streptolysin $\mathrm{O}$ but not streptolysin S.

The haemolytic activity of the group $\mathrm{G}$ clinical isolate differed from that of the group $\mathrm{C}$ isolates in that $\beta$ haemolysis was observed on all five media when incubated anaerobically. However, as observed with the group $\mathrm{C}$ isolates, the group $\mathrm{G}$ strain was $\beta$-haemolytic

Table 1. Detection of $\beta$-haemolytic Lancefield group A, C and $\mathrm{G}$ streptococci in pharyngeal cultures when grown on CNA-P and other blood agar media

\begin{tabular}{lrrrr}
\hline & \multicolumn{4}{c}{$\begin{array}{c}\text { Number (\%) of 361 pharyngeal cultures positive for } \\
\beta \text {-haemolytic }\end{array}$} \\
\cline { 2 - 5 } streptococci of Lancefield group
\end{tabular}


Table 2. Effect of blood agar medium composition and incubation atmosphere on haemolytic activity of streptococcal cultures

\begin{tabular}{|c|c|c|c|c|c|c|c|c|c|c|c|}
\hline \multirow{3}{*}{$\begin{array}{l}\text { Culture } \\
\text { medium }\end{array}$} & \multirow{3}{*}{$\begin{array}{l}\text { Incubation } \\
\text { atmosphere }\end{array}$} & \multicolumn{10}{|c|}{ Presence of $\beta$-haemolysis and terminal $\mathrm{pH}$ of cultures* } \\
\hline & & \multicolumn{2}{|c|}{ Group C strains ${ }^{\dagger}$} & \multicolumn{2}{|c|}{$\begin{array}{c}\text { Group G } \\
\text { strain } 0662\end{array}$} & \multicolumn{2}{|c|}{$\begin{array}{c}\text { S. pyogenes } \\
\text { strain 82-541 } \\
\text { (SLS-negative) }\end{array}$} & \multicolumn{2}{|c|}{$\begin{array}{c}\text { S. dysgalactiae } \\
\text { subsp. } \\
\text { dysgalactiae } \\
\text { strain } 67\end{array}$} & \multicolumn{2}{|c|}{$\begin{array}{c}\text { S. equi subsp. } \\
\text { zooepidemicus } \\
\text { strain } 4881\end{array}$} \\
\hline & & $\beta$ & $\mathrm{pH}$ & $\beta$ & $\mathrm{pH}$ & $\beta$ & $\mathrm{pH}$ & $\beta$ & $\mathrm{pH}$ & $\beta$ & $\mathrm{pH}$ \\
\hline \multirow[t]{3}{*}{ CNA-P } & Anaerobic & + & 7.3 & + & 7.4 & + & 7.2 & - & 7.2 & + & 7.2 \\
\hline & $\mathrm{CO}_{2} 5 \%$ & + & 7.3 & + & 7.3 & + & 7.3 & - & 7.2 & + & 7.4 \\
\hline & Aerobic & + & 7.4 & + & 7.3 & + weak & 7.3 & - & 7.3 & + & 7.4 \\
\hline \multirow[t]{3}{*}{ CNA } & Anaerobic & - & 6.1 & + & 6.7 & - & 5.9 & - & 6.0 & + & 6.6 \\
\hline & $\mathrm{CO}_{2} \quad 5 \%$ & - & 6.4 & - & 6.8 & - & 6.5 & - & 6.6 & + & 6.6 \\
\hline & Aerobic & - & 6.6 & - & 6.8 & - & 6.5 & - & 6.5 & + & 6.8 \\
\hline \multirow[t]{3}{*}{$\mathrm{BA}-\mathrm{Ca}$} & Anaerobic & $+1-\dot{t}$ & $6.7-6.9$ & + & 7.0 & - & 6.7 & - & 6.6 & + & 6.8 \\
\hline & $\mathrm{CO}_{2} \quad 5 \%$ & + weak & 7.0 & + & 7.2 & - & 6.9 & - & 7.0 & + & 6.9 \\
\hline & Aerobic & + weak & 7.1 & + & 7.2 & - & 7.0 & - & 7.0 & + & 6.9 \\
\hline \multirow[t]{3}{*}{ SBA } & Anaerobic & - & 6.5 & + & 6.4 & - & 6.4 & - & 6.7 & + & 6.4 \\
\hline & $\mathrm{CO}_{2} \quad 5 \%$ & - & 6.8 & - & 6.5 & - & 6.8 & - & 6.9 & + & 6.7 \\
\hline & Aerobic & - & 6.9 & - & 6.6 & - & 6.8 & - & 6.9 & + & 6.9 \\
\hline \multirow[t]{3}{*}{$\mathrm{EH}$} & Anaerobic & - & 6.0 & + & 6.2 & - & 5.9 & - & 5.7 & + & 5.9 \\
\hline & $\mathrm{CO}_{2} \quad 5 \%$ & - & 6.2 & - & 6.5 & - & 6.3 & - & 6.1 & + & 6.1 \\
\hline & Aerobic & - & 6.2 & - & 6.4 & - & 6.2 & - & 6.1 & + & 6.3 \\
\hline
\end{tabular}

* Terminal $\mathrm{pH}$ in the confluent zone and presence or absence of $\beta$-haemolysis on each medium was noted.

Group C strains $0726,1446,3610$ and 1312 gave identical results except as noted below and for convenience are recorded as a composite. ${ }^{*}$ At $\mathrm{pH}$ values of $<6.9, \beta$-haemolysis was not observed.

in all atmospheres when grown on CNA-P or on BA-Ca (terminal $\mathrm{pH}$ range 7.0-7.2) and it was non-haemolytic on CNA, SBA and EH media when cultured in an aerobic or $\mathrm{CO}_{2}$-enriched atmosphere.

S. equi subsp. zooepidemicus 4881 (a $\beta$-haemolytic group $\mathrm{C}$ animal isolate) was strongly $\beta$-haemolytic on all five media tested under the three different atmospheric conditions. The largest haemolytic zones were produced on $\mathrm{EH}$ medium. The terminal $\mathrm{pH}$ ranged from a low of 5.9 on $\mathrm{EH}$ under anaerobic conditions to a high of $\mathrm{pH} 7.4$ on CNA-P under $\mathrm{CO}_{2} \quad 5 \%$. $S$. dysgalactiae subsp. dysgalactiae strain 67 (nonhaemolytic group $\mathrm{C}$ animal isolate) was generally nonhaemolytic, but did produce some $\alpha$-haemolysis under a few test conditions within the confluent growth zones of these cultures.

Strains of $S$. pyogenes known to be deficient in production of either streptolysin $\mathrm{O}$ or streptolysin $\mathrm{S}$ were also tested for their haemolytic activity. $S$. pyogenes strain $82-541$ has a $T n 916$ insertion that abolishes streptolysin $\mathrm{S}$ activity and it only produces streptolysin $\mathrm{O}$. The strain is $\beta$-haemolytic on CNA-P under anaerobic and $\mathrm{CO}_{2} 5 \%$ incubation, but only has a small inner $\beta$ - and wide outer $\alpha$-haemolytic zone on CNA-P when incubated in air. No haemolysis was observed on any of the other media under the conditions tested. The Blackmore strain (SLO mutant) and wild-type M-type 1 strains were $\beta$-haemolytic under all test conditions, with activity and terminal $\mathrm{pH}$ values similar to those produced by $S$. equi subsp. zooepdemicus 4881 (data not shown). Strain Blackmore displayed enhanced zones of haemolysis on $\mathrm{EH}$ medium comparable to zones observed with wild-type $S$. pyogenes and $S$. equi subsp. zooepidemicus.

These results suggested that the enhanced haemolysis observed on CNA-P was due to increased streptolysin $\mathrm{O}$ activity, whereas the increased haemolysis on $\mathrm{EH}$ agar was streptolysin S-dependent. To confirm that the CNA-P-specific haemolytic activity of the streptococci isolated in the present study was due to streptolysin $\mathrm{O}$, the strains were plated on CNA-P containing cholesterol. Cholesterol and related sterols with a $\beta$-hydroxyl group on carbon 3 bind to SLO and prevent its absorption to susceptible cell membranes [30]. In the presence of cholesterol, $\beta$-haemolysis by the group $\mathrm{C}$ and $\mathrm{G}$ clinical isolates and by $S$. pyogenes $82-541$ was abolished, whereas the haemolysis by $S$. pyogenes Mtype 1 and $S$. equi subsp. zooepidemicus 4881 was unaffected.

Human but not animal isolates of group $C$ and $G$ streptococci have been shown to contain emm-like genes $(\mathrm{emmL})$ that encode $\mathrm{M}$ proteins with biological and structural features similar to those observed for $\mathrm{M}$ proteins found in $S$. pyogenes [31,32]. The group $\mathrm{C}$ and $\mathrm{G}$ isolates were tested for the presence of an $e m m \mathrm{~L}$ gene by PCR with $S$. pyogenes-specific primers. All five isolates yielded a product c. $1300-1350 \mathrm{bp}$, whereas $S$. dysgalactiae subsp. dysgalactiae and $S$. equi subsp. zooepidemicus were PCR negative. Three of the four group C streptococcal PCR amplicons had identical HaeIII/HincII digest products of $1000 \mathrm{bp}$ and $350 \mathrm{bp}$ (designated 1446) and the fourth isolate (0726) had products of $1000 \mathrm{bp}$ and $300 \mathrm{bp}$. The group G streptococcal PCR amplicon did not cut with Hae III 
and HincII restriction endonucleases. The $5^{\prime}$ ends of the three unique PCR products were sequenced and BLAST searches were conducted. The 1446 and 0726 sequences were identical to each other through the $450 \mathrm{bp}$ sequenced, with the exception of an additional $42 \mathrm{bp}$ located within the first $200 \mathrm{bp}$ of the 1446 sequence. None of the three sequences showed significant homology to sequences in the emm database (Centers for Disease Control and Prevention, Atlanta). The 0726 and 1446 sequences did not match any GenBank sequences. Interestingly, the highest homology of the group G 0662 sequence was to a novel emmtype sequence from a putative $S$. pyogenes strain isolated recently in Thailand (GenBank AF104408). Species indentification methods were not reported in the GenBank entry. In light of the recent report by Brandt et al. [20] of isolates of $S$. dysgalactiae subsp. equisimilis possessing the Lancefield group A antigen, classification by serogrouping should be viewed with caution if used as the sole method for identification. The three unique partial emm sequences have been entered in GenBank with the accession numbers AF226694 (strain 0726), AF226695 (strain 1446) and AF226696 (strain 0662).

Comparative analysis of partial 16S rRNA sequences of Streptococcus species by Bently et al. [16] revealed a region of sequence heterogeneity (c. bp 145-300 of the Escherichia coli numbering system) which allowed species discrimination and an assignment of intrageneric relationships. The $5^{\prime}$ half of the $16 \mathrm{~S}$ DNA was amplified and sequenced with conserved eubacterial 16S PCR primers. The sequence obtained from the group $\mathrm{G}$ isolate matched the $16 \mathrm{~S}$ sequence of $S$. dysgalactiae subsp. equisimilis (GenBank AB008926) as did the sequences from the four group $\mathrm{C}$ isolates, except for a $\mathrm{C} / \mathrm{T}$ transversion at bp 218 with respect to the GenBank sequence that was conserved in all four isolates.

\section{Discussion}

CNA-P was originally developed in this laboratory to minimise or abolish the bacteriocin activity of some strains of $S$. salivarius in order to enhance the detection of $S$. pyogenes when these are present in small numbers as in the carrier state [24]. On traditional isolation media, the growth of $\beta$-haemolytic streptococci is inhibited in the vicinity of bacteriocinproducing $S$. salivarius and bacteriocin-sensitive streptococci will only form characteristic colonies if they are outside the zone of influence of bacteriocin producers. In the course of the evaluation of CNA-P in a clinical laboratory setting, it was noted that in some specimens $\beta$-haemolytic colonies were isolated only on CNA-P irrespective of the concomitant presence of bacteriocin-producing $S$. saliviarius. The previous observation that streptolysin S-deficient $S$. pyogenes produced $\beta$-haemolysis on CNA-P but not on SBA [24] led to the suspicion that these isolates may also be SLS-deficient. During the short duration of this trial, seven group $\mathrm{A}, \mathrm{C}$ or $\mathrm{G}$ streptococci were isolated that were not $\beta$-haemolytic on conventional blood agar media. The high frequency of pharyngitisassociated specimens that yielded these haemolysin variants and the high proportion of the streptococcal population that they represented in these specimens indicated that these bacteria could have considerable clinical significance.

The elimination of the $\beta$-haemolytic activity of the variant strains on CNA-P by addition of cholesterol to the medium suggested that streptolysin $\mathrm{O}$ was the only haemolysin produced by these strains. Variant strains were isolated in high numbers as the predominant organism in clinical samples from patients suffering symptoms of pharyngitis, which suggested a possible aetiological association with the disease. However, as it was not possible to access patient records or to obtain follow-up specimens during the course of the present study the possibility that these bacteria were sometimes carried in large numbers as commensals could not be excluded. Also, due to the short duration of the trial and the relatively small number of samples processed, it was not possible to predict whether variants would generally be present in such a high proportion of specimens or if these isolates (particularly those of group C) were representative of a localised outbreak.

A few reports in the very early literature on streptococci documented the occurrence of non-haemolytic variants of $S$. pyogenes [33-35]. Associations of non-haemolytic variants with disease outbreaks were reported by Coburn and Pauli [36] and Colebrook et al. [37]. Both episodes were caused by M-type 12 variants [22]. The strains isolated by Colebrook et al. produced only streptolysin O. More recently, James and McFarland (1971) reported an outbreak involving 118 air force recruits in Colorado, with six subsequent cases of rheumatic fever [23]. Interestingly, James and McFarland commented that although Colebrook et al. had speculated in 1942 that pathogenic streptococci were being missed in puerperal or surgical infections because of variations in haemolysin production, no further mention of non-haemolytic variants had appeared in the subsequent literature until their own report in 1971. Clearly, the question of the significance of these haemolysin variants as infectious disease agents has not been addressed adequately yet. With more widespread application of streptolysin O-enhancing media such as CNA-P the extent of their distribution in disease and carrier associations should be clarified.

This research was supported by the Thrasher Foundation, the National Heart Foundation of New Zealand, the Community Trust of Otago and the Health Research Council of New Zealand. We thank Janet Wilson and Nadika Liyanarachchy of Southern Community Laboratories for their assistance with this study and in particular to Megan Inglis and Nancy Ragland for technical assistance. 


\section{References}

1. Bradley SF, Gordon JJ, Baumgartner DD, Marasco WA, Kauffman CA. Group C streptococcal bacteremia: analysis of 88 cases. Rev Infect Dis 1991; 13: 270-280.

2. Carmeli Y, Ruoff KL. Report of cases of and taxonomic considerations for large-colony-forming Lancefield group C streptococcal bacteremia. $J$ Clin Microbiol 1995; 33 2114-2117.

3. Efstratiou A. Outbreaks of human infection caused by pyogenic streptococci of Lancefield groups C and G. J Med Microbiol 1989; 29: 207-219.

4. Salata RA, Lerner PI, Shlaes DM, Gopalakrishna KV, Wolinsky E. Infections due to Lancefield group $\mathrm{C}$ streptococci. Medicine (Baltimore) 1989; 68: 225-239.

5. Gnann JW, Gray BM, Griffin FM, Dismukes WE. Acute glomerulonephritis following group $\mathrm{G}$ streptococcal infection. J Infect Dis 1987; 156: 411-412.

6. Deighton C. Beta haemolytic streptococci and reactive arthritis in adults. Ann Rheum Dis 1993; 52: 475-482.

7. Jansen TLTA, Janssen M, de Jong AJL. Reactive arthritis associated with group $\mathrm{C}$ and group $\mathrm{G}$ beta-hemolytic streptococci. J Rheumatol 1998; 25: 1126-1130.

8. Baumgarten A, von Graevenitz A, Greco T. Serological evidence for a causative role of non-group A hemolytic streptococci in pharyngitis. Zentralbl Bakteriol Mikrobiol Hyg [A] 1981; 249: 460-465.

9. Cohen D, Ferne M, Rouach T, Bergner-Rabinowitz S. Foodborne outbreak of group $G$ streptococcal sore throat in an Israeli military base. Epidemiol Infect 1987; 99: 249-255.

10. Corson AP, Garagusi VF, Chretien JH. Group C beta-hemolytic streptococci causing pharyngitis and scarlet fever. South Med J 1989; 82: 1119-1121.

11. Fox K, Turner J, Fox A. Role of beta-hemolytic group C streptococci in pharyngitis: incidence and biochemical characteristics of Streptococcus equisimilis and Streptococcus anginosus in patients and healthy controls. J Clin Microbiol 1993; 31: $804-807$.

12. Martin NJ, Kaplan EL, Gerber MA et al. Comparison of epidemic and endemic group $\mathrm{G}$ streptococci by restriction enzyme analysis. J Clin Microbiol 1990; 28: 1881-1886.

13. Turner JC, Hayden FG, Lobo MC, Ramirez CE, Murren D. Epidemiologic evidence for Lancefield group C beta-hemolytic streptococci as a cause of exudative pharyngitis in college students. J Clin Microbiol 1998; 35: 1-4.

14. Chowdhury MNH, Kambal AM, Al-Eissa YA, Khaliq MRA, Al-Ayed IH, Al-SanieAM. Non-group A streptococci: are they pathogens in the throat? $J R$ Soc Health 1997; 117: 160-163.

15. Cimolai NB, Morrison BJ, MacCulloch L, Smith DF, Hlady J. Beta-haemolytic non-group A streptococci and pharyngitis: a case-control study. Eur J Pediatr 1991; 150: 776-779.

16. Bently RW, Leigh JA, Collins MD. Intrageneric structure of Streptococcus based on comparative analysis of small-subunit rRNA sequences. Int J Syst Bacteriol 1991; 41: 487-494.

17. Bert F, Branger C, Poutrel B, Lambert-Zechovsky N. Differentiation of human and animal strains of Streptococcus dysgalactiae by pulsed-field gel electrophoresis. FEMS Microbiol Lett 1997; 150: 107-112.

18. Vandamme P, Bot B, Falsen E, Kersters K, Devriese LA. Taxonomic study of Lancefield streptococcal groups C, G, and L (Streptococcus dysgalactiae) and proposal of $S$. dysgalactiae subsp. equisimilis subsp. nov. Int J Syst Bacteriol 1996; 46: 774-781.
19. Vieira VV, Teixeira LM, Zahner V et al. Genetic relationships among the different phenotypes of Streptococcus dysgalactiae strains. Int J Syst Bacteriol 1998; 48: 1231-1243.

20. Brandt CM, Haase G, Schnitzler N, Zbinden R, Lütticken R. Characterization of blood culture isolates of Streptococcus dysgalactiae subsp. equisimilis possessing Lancefield's Group A antigen. J Clin Microbiol 1999; 37: 4194-4197.

21. Bert F, Lambert-Zechovsky N. Analysis of a case of recurrent bacteraemia due to group A Streptococcus equisimilis by pulsed-field gel electrophoresis. Infection 1997; 25: 250-251.

22. Rammelkamp CH, Dingle JH. Pathogenic streptococci. Annu Rev Microbiol 1948; 2: 279-304.

23. James L, McFarland RB. An epidemic of pharyngitis due to nonhemolytic group A streptococcus at Lowry Air Force Base. $N$ Engl J Med 1971; 284: 750-752.

24. Dierksen KP, Ragland NL, Tagg JR. A new alkaline pHadjusted medium enhances detection of $\beta$-hemolytic streptococci by minimising bacterial interference due to Streptococcus salivarius. J Clin Microbiol 2000; 38: 643-650.

25. Tagg JR, Vugler LG. Enhancement of the hemolytic activity of group B streptococci and streptolysin S-deficient group A streptococci on blood agar medium containing staphylococcal beta-lysin. J Microbiol Methods 1986; 5: 215-220.

26. Johnson DR, Kaplan EL, Sramek J et al. Laboratory diagnosis of group A streptococcal infections. Geneva, World Health Organization. 1996.

27. Beall B, Facklam R, Thompson T. Sequencing emm-specific PCR products for routine and accurate typing of group A streptococci. J Clin Microbiol 1996; 34: 953-958.

28. Wilson $\mathrm{KH}$, Blitchington RB, Greene RC. Amplification of bacterial $16 \mathrm{~S}$ ribosomal DNA with polymerase chain reaction. $J$ Clin Microbiol 1990; 28: 1942-1946.

29. Whatmore AM, Kapur V, Sullivan DJ, Musser JM, Kehoe MA. Non-congruent relationships between variation in emm gene sequences and the population genetic structure of group A streptococci. Mol Microbiol 1994; 14: 619-631.

30. Alouf JE. Streptococcal toxins (streptolysin O, streptolysin S, erythrogenic toxin). Pharmacol Ther 1980; 11: 661-717.

31. Schnitzler N, Podbielski A, Baumgarten G, Mignon M, Kaufhold A. M or M-like protein gene polymorphisms in human group G streptococci. J Clin Microbiol 1995; 33: $356-363$.

32. Bisno AL, Collins CM, Turner JC. M proteins of group C streptococci isolated from patients with acute pharyngitis. $J$ Clin Microbiol 1996; 34: 2511-2515.

33. Cumming WM. On the pseudohaemolytic streptococci isolated from the sputum in pulmonary tuberculosis. J Pathol Bacteriol 1927; 30: 279-300.

34. Todd EW. The conversion of hemolytic streptococci to nonhemolytic forms. J Exp Med 1928; 48: 493-511.

35. Lancefield RC. Loss of the properties of hemolysin and pigment formation without change in immunological specificity in a strain of Streptococcus haemolyticus. J Exp Med 1934; 59: 459-469.

36. Coburn AF, Pauli RH. The interaction of host and bacterium in the development of communicability by Streptococcus haemolyticus. J Exp Med 1941; 73: 551-570.

37. Colebrook L, Elliot SD, Maxted WR, Morley CW, Mortell M. Infection by non-haemolytic group-A streptococci. Lancet 1942; 2: 30-31. 